\title{
Entropy and Wigner Functions
}

\author{
G. Manfredi ${ }^{1, *}$ and M. R. Feix ${ }^{2}$ \\ ${ }^{1}$ Laboratoire de Physique des Milieux Ionisés, Université Henri Poincaré, \\ BP239, 54506 Vandoeuvre-les-Nancy, France \\ ${ }^{2}$ Subatech, Ecole des Mines de Nantes, BP 20722, 44307 Nantes Cedex 3, France \\ * Electronic address : giovanni.manfredi@lpmi.uhp-nancy.fr
}

\begin{abstract}
The properties of an alternative definition of quantum entropy, based on Wigner functions, are discussed. Such definition emerges naturally from the Wigner representation of quantum mechanics, and can easily quantify the amount of entanglement of a quantum state. It is shown that smoothing of the Wigner function induces an increase in entropy. This fact is used to derive some simple rules to construct positive definite probability distributions which are also admissible Wigner functions.
\end{abstract}

PACS : 03.65.-w, 05.30.-d

\section{Introduction}

Entropy is the central concept of thermodynamics and statistical mechanics. It was introduced by Clausius in the mid-19th century as a phenomenological variable that quantifies the intrinsic irreversibilty of thermodynamical processes. It was Boltzmann who recognized the link between entropy and the lack of information about a system, defined as the number $\Gamma$ of microstates which have the same macroscopic properties. The celebrated formula

$$
S_{\mathrm{B}}=k_{\mathrm{B}} \ln \Gamma
$$

establishes such a link in a mathematically rigorous manner (in the rest of this article we shall use units for which $k_{\mathrm{B}}=1$ : with this prescription, entropy becomes a dimensionless quantity). Boltzmann, of course, derived this formula in the context of classical statistical mechanics. In classical physics, microstates are defined as points in a continuous $2 D$-dimensional phase space ( $D$ is the number of degrees of freedom of the system under consideration), and cannot be "counted" in any meaningful sense. Therefore, Boltzmann took as the number $\Gamma$ of microstates the available volume in phase space $\Omega$ divided by the volume of a unit cell (unspecified at the time when Boltzmann published his work, but which will turn out to be Planck's constant,raised to the appropriate power, $\left.h^{D}\right): \Gamma=\Omega / h^{D}$. In quantum mechanics, a 
microstate is described by a wave function, which contains all the information about the state of the system. In contrast to the classical case, now there is no ambiguity, since quantum states are discrete in principle. Hence, although the macrostate has a huge number of possible microstates consistent with it, this number, $\Gamma$, is nevertheless definite and finite.

The most general quantum system is described by a density matrix, i.e. a positive-definite, Hermitian operator, with unit trace. In terms of the density matrix $\rho$, the entropy can be expressed in the following way, due to Von Neumann [1]

$$
S_{\mathrm{VN}}=-\operatorname{Tr} \rho \ln \rho .
$$

This is the standard definition of entropy, which generalizes Boltzmann's expression to quantum mechanics. Although unambiguously defined, however, $S_{\mathrm{VN}}$ can be extremely difficult to compute in practice, since one would need to diagonalize $\rho$ in order to compute the trace of its logarithm. Von Neumann's entropy (VN) has a number of good properties, which will be detailed in the following sections. Here we note that, if $\alpha_{i} \geq 0$ are the eigenvalues of the density matrix $\left(\sum_{i} \alpha_{i}=1\right)$, the $\mathrm{VN}$ entropy becomes $S_{\mathrm{VN}}=-\sum_{i} \alpha_{i} \ln \alpha_{i}$. Therefore $S_{\mathrm{VN}} \geq 0$, and the equality holds only if we have complete information, i.e. if only one of the eigenvalues is different from zero: in this case, the system is in the pure state corresponding to this eigenvalue. Another crucial property of $S_{\mathrm{VN}}$ is that it is conserved as $\rho$ evolves according to the quantum Liouville equation

$$
i \hbar \frac{\partial \rho}{\partial t}=H \rho-\rho H
$$

where $H$ is the Hamiltonian. Indeed, the trace of any functional $F$ of the density matrix $\operatorname{Tr} F(\rho)$ is also conserved. This fact can be used to define other entropy-like quantities. Not all this quantities are equivalent, however, and we will show in the following section that only one of them is particularly adapted to the Wigner representation of quantum mechanics.

The classical limit of the Von Neumann entropy, Eq. (2), is obtained by replacing the density matrix with the phase space probability distribution $f(x, p)$ (for simplicity, we will consider systems with only one degree of freedom, $D=1$ ), and the trace with the integral in phase space. One obtains the following expression, due to Gibbs

$$
S_{\mathrm{CL}}=-\int f \ln (f h) d x d p,
$$

and the probability distribution is positive and normalized to unity. Note that the classical entropy is defined up to an additive constant, which means that the constant $h$ in the argument of the logarithm in Eq. (4) can be chosen arbitrarily, although it seems reasonable to use Planck's constant $h=2 \pi \hbar$. Indeed, if $f$ is constant inside a certain phase space volume $\Omega$ and zero elsewhere (i.e. at thermodynamic equilibrium), then $S_{\mathrm{CL}}=\ln (\Omega / h)$, in agreement with Boltzmann's original definition, Eq. (11). We also stress that $S_{\mathrm{CL}}$ can take negative values, in contrast with $S_{\mathrm{VN}}$, which is always non-negative. From the previous discussion, it is easy to conclude that $S_{\mathrm{CL}}$ will be negative when $\Omega<h$. This means that we are trying to localize a particle on a phase space region smaller than Planck's constant, and therefore violate the uncertainty principle. For probability distributions that satisfy the uncertainty principle, the classical entropy is positive. Similarly to the quantum mechanical case, the classical entropy is conserved for a Hamiltonian process, i.e. when the probability distribution evolves according to the classical Liouville equation. Again, the phase space integral of 
any functional $F(f)$ is also conserved (indeed, $f$ itself is conserved, since it is just transported along the classical trajectories).

In this paper, we discuss the properties of an alternative definition of quantum entropy, based on Wigner functions. Although this entropy has already been known for some time (generally expressed in terms of the density matrix), we feel that its properties are not fully appreciated. In particular, it will be shown that such a definition of entropy emerges naturally from the Wigner representation of quantum mechanics. It has therefore a privileged status compared to the many other definitions proposed in the literature, and deserves to be studied in some depth.

The Wigner representation [2] is a useful tool to express quantum mechanics in a phase space formalism (for reviews see [3, 4). Although it was derived by Wigner for technical purposes, this approach has recently attracted much interest, since it is well-suited to analyze the transition from classical to quantum dynamics. The Wigner representation can deal with both pure and mixed quantum states, and is completely equivalent to the more usual picture based on the density matrix. In this representation, a quantum state is described by a Wigner function (i.e. a function of the phase space variables - see next Section), and the Wigner equation provides an evolution equation for the state which is equivalent to the quantum Liouville equation (3). It will be shown that, if one tries to define an entropy functional in the framework of Wigner's representation, only one 'reasonable' choice is possible, and this is discussed in the next Section. Subsequently, we will discuss the properties of such an entropy (Sec. III), and present some examples of its applications in Secs. IV and V.

\section{Quantum Entropy}

The quantum distribution function $W(x, p)$ is defined in terms of the density matrix $\rho(x, y)$ for a quantum mixed state

$$
W(x, p)=\frac{1}{2 \pi \hbar} \int \rho\left(x-\frac{\lambda}{2}, x+\frac{\lambda}{2}\right) \exp \left(\frac{i p \lambda}{\hbar}\right) d \lambda,
$$

or in terms of the wavefunction $\psi(x)$ for a pure state

$$
W(x, p)=\frac{1}{2 \pi \hbar} \int \psi\left(x-\frac{\lambda}{2}\right) \psi^{*}\left(x+\frac{\lambda}{2}\right) \exp \left(\frac{i p \lambda}{\hbar}\right) d \lambda .
$$

The function $W(x, p)$ possesses many of the properties of a phase space probability distribution: it is real, normalized to unity, and, when integrated over $x$ or $p$, gives the correct marginal distribution, e.g. $\int W d p=\rho(x, x)=$ spatial density. Furthermore, it can be used to compute averages of any dynamical variable $A(x, p):\langle A\rangle=\int W A d x d p$. Note however that, since some terms in $A(x, p)$ may not commute, it is necessary to establish a non-ambiguous correspondence between classical variables and quantum operators (Weyl's rule) 甮. Despite these good properties, the Wigner function cannot be interpreted as a probability distribution, since it can assume negative values. The only pure state whose Wigner function is positive definite is given by the minimum uncertainty packet (i.e. a Gaussian wavefunction).

The evolution of $W(x, p, t)$ is governed by the Wigner equation, which replaces the classical Liouville equation :

$$
\frac{\partial W}{\partial t}+\frac{p}{m} \frac{\partial W}{\partial x}=\frac{i}{2 \pi \hbar^{2}} \int\left[\Phi\left(x-\frac{z}{2}\right)-\Phi\left(x+\frac{z}{2}\right)\right] \exp \left(-\frac{i}{\hbar}\left(p-p^{\prime}\right) z\right) W\left(x, p^{\prime}, t\right) d z d p^{\prime},
$$


where $\Phi(x)$ is the potential. The Wigner equation is equivalent to the quantum Liouville equation (3), and can describe the evolution of both pure states and mixtures. However, in the present work, we shall privilege the Wigner formalism over the density matrix one, since it is easier to represent in the classical phase space, and it allows a more staightforward treatment of the semi-classical limit.

We would like to define an entropy functional in terms of Wigner functions. The classical choice, Eq. (雨), obviously cannot work, since $W$ can assume negative values. It is easy to show the existence of two simple functionals of $W$ that are invariant under Eq. (7)): the first is the total probability $\int W d x d p=1$; the second invariant is $\int W^{2} d x d p$, which has no obvious physical meaning. We stress that this is a property of Eq. (7), and does not depend on whether $W$ represents a pure state, a mixture, or even a state which violates the uncertainty principle. However, the fact that the latter expression is indeed invariant, suggests that we introduce the following definition of entropy

$$
S_{2}=1-(2 \pi \hbar)^{D} \int W^{2} d x d p
$$

where $D$ is the number of degrees of freedom: except where otherwise stated, we will always work with systems for which $D=1$.

The $S_{2}$ entropy can be expressed in terms of the density matrix $\rho$

$$
S_{2}=1-\operatorname{Tr} \rho^{2}
$$

a result which follows from the fact that $W$ is related to the Fourier transform of $\rho$. Equation (9) has been used in the literature as an entropy-like quantity [5], and sometimes referred to as the linear entropy. Its relevance to Wigner functions has been noticed by some authors [6], but its full implications have not, to our knowledge, been appreciated and developed. We first notice that this is the only expression of entropy having the same functional form when expressed in terms of either $W$ or $\rho$ (for example, $\int W^{4}$ is not simply related to $\operatorname{Tr} \rho^{4}$ ). Secondly, and most importantly, the very structure of Wigner's equation selects the functional $S_{2}$ as a special candidate for a definition of entropy. It is therefore important to study its properties and implications.

When $W$ is an admissible Wigner function (i.e. when it represents either a pure or a mixed quantum state), the previous entropy satisfies the relation $0 \leq S_{2} \leq 1$, and $S_{2}=0$ holds for a pure state, which is a reasonable result, since pure states contain the maximum information available. Indeed, it is possible to define quantum information as the complement of $S_{2}$ to unity, $I=1-S_{2}$. Note that $S_{2}$ can become negative only for states that violate the uncertainty principle, as it will be explained in Sec. III. We point out that $S_{2}=0$ is a necessary, but definitely not sufficient condition for the corresponding Wigner function to represent a pure state [3]. This can be shown by finding a counter-example. Let us define the Wigner function as $W=\sum_{i=1}^{3} \alpha_{i} W_{i}$, where the $W_{i}$ are orthogonal pure states, and $\alpha_{1}=\alpha_{2}=2 / 3, \alpha_{3}=-1 / 3$. Even though the coefficients $\alpha_{i}$ sum up to unity, $W$ does not represent an admissible Wigner function, since one of the coefficients (which represent probabilities) is negative. However, it is simple to prove that $S_{2}[W]=0$. Incidentally, this example has shown the existence of phase space functions which represent neither pure states nor mixtures. This point will be discussed in more detail in the next Section. 
This entropy is related to a formula proposed by Tsallis [7], which has stimulated much work in the last decade (see, for example, [8] and references therein). If $\left\{\alpha_{i}\right\}$ is a set of probabilities adding up to unity, Tsallis entropy is defined by

$$
S_{q}=\frac{1-\sum_{i} \alpha_{i}^{q}}{q-1}
$$

where $q$ is a real, not necessarily positive, number, and the standard entropy is recovered for $q \rightarrow 1$. Tsallis entropy is a possible, and indeed useful, way to generalize the Boltzmann-Von Neumann expression, and has been employed by several authors to study the thermodynamics of strongly correlated systems, such as self-gravitating gases and inviscid fluids [8].

Equation (8), is the continuous counterpart of the discrete Tsallis entropy with $q=2$. The continuous formula can be recovered by the following heuristic argument. Let us cover the phase space with cells of size $\Delta x \Delta p$. The discrete probabilities are then $\alpha_{i}=W\left(x_{i}, p_{i}\right) \Delta x \Delta p$, and the discrete entropy becomes

$$
S_{2}=1-\Delta x \Delta p \sum_{i} W^{2}\left(x_{i}, p_{i}\right) \Delta x \Delta p
$$

The sum in Eq. (11) gives the integral $\int W^{2} d x d p$. However, we cannot let the factor $\Delta x \Delta p$ in front of the sum go to zero, since this would violate the uncertainty relation. Indeed, we obtain the correct continuous formula [Eq. (8) with $D=1$ ] by taking for $\Delta x \Delta p$ the smallest value allowed by quantum mechanics, i.e. Planck's constant $h=2 \pi \hbar$.

Another way to go from the continuous to the discrete formula, is to consider a Wigner function that is the sum of $N$ orthogonal pure states $W(x, p)=\sum_{i=1}^{N} \alpha_{i} W_{i}(x, p)$. Of course $W$ represents a quantum mixture. We recall the following useful relation, valid for orthogonal pure states:

$$
\int W_{i} W_{j} d x d p=\delta_{i j} / 2 \pi \hbar
$$

where $\delta_{i j}$ is the Kronecker delta. By developing $W$ in terms of the $W_{i}$ in Eq. (8), and making use of Eq. (12), we obtain Tsallis discrete entropy $S_{2}=1-\sum_{i=1}^{N} \alpha_{i}^{2}$. We stress again that the above properties are valid for the quadratic entropy $S_{2}$, but do not hold for other functionals involving higher powers of $W$.

It is interesting to show that a local entropy $\sigma$ and an entropy flux $J_{S}$ can also be defined:

$$
\sigma(x, t)=\int W d p-2 \pi \hbar \int W^{2} d p, \quad J_{S}(x, t)=\int \frac{p}{m} W d p-2 \pi \hbar \int \frac{p}{m} W^{2} d p .
$$

Of course one has $S_{2}=\int \sigma d x$. By multiplying Eq. (7) by $W$ and integrating over momentum space, one can prove that the local entropy obeys a continuity equation :

$$
\frac{\partial \sigma}{\partial t}+\frac{\partial J_{S}}{\partial x}=0
$$

which shows that entropy can be transfered from one spatial location to another, but is globally conserved. The physical meaning of $\sigma$ is easier to grasp if we express it in terms of the density matrix in the position representation. With the help of Eq. (5) one finds (we drop the time dependence)

$$
\sigma(x)=\rho(x, x)-\int|\rho(x-\lambda / 2, x+\lambda / 2)|^{2} d \lambda .
$$


Equation (15) shows that entropy is closely related to the off-diagonal terms of the density matrix. For a pure state, $\rho(x, y)=\psi(x) \psi^{\star}(y)$ ( $\psi$ is the wavefunction), and the local entropy can be expressed in terms of the spatial density $n(x)=|\psi(x)|^{2}=\rho(x, x)$

$$
\sigma(x)=n(x)-\int n\left(x-\frac{\lambda}{2}\right) n\left(x+\frac{\lambda}{2}\right) d \lambda \equiv n(x)-\iota(x),
$$

where we have defined the local quantum information $\iota(x)$ so that $I=\int \iota d x$. It appears that $\iota(x)$ is a density autocorrelation function, which shows that, in quantum mechanics, information and spatial correlations are intimately close concepts.

\section{Properties of Quantum Entropy}

The expression given in Eq. (8) has proven to be a fruitful tool to quantify some key properties of quantum systems, such as nonlocal correlations. In order to be an appropriate definition of entropy it should nevertheless satisfy some standard properties [9], among which concavity and additivity are particularly fundamental. Some of these properties were previously studied by Tsallis [7] for the discrete case.

1. Concavity. This means that, if $W=\sum_{i=1}^{N} \alpha_{i} W_{i}$ (where the $W_{i}$ are not necessarily pure orthogonal states), then the following inequality holds

$$
S_{2}[W] \geq \sum_{i=1}^{N} \alpha_{i} S_{2}\left[W_{i}\right] .
$$

The proof is obtained by direct calculation for $N=2$, and then is easily extended to higher $N$ by recursive arguments.

Note that we can also prove an upper bound for $S_{2}$

$$
S_{2}[W] \leq \sum_{i=1}^{N} \alpha_{i}^{2} S_{2}\left[W_{i}\right]+1-\sum_{i=1}^{N} \alpha_{i}^{2}
$$

which holds for $W_{i}$ representing both pure states or mixtures. The term $1-\sum_{i} \alpha_{i}^{2}$ represent the so-called mixing entropy. The proof of Eq. (18) relies on the following inequality [3]

$$
\int W_{i} W_{j} d x d p \geq 0
$$

which is valid for all admissible Wigner functions, pure or mixed states (see Sec. IV for a definition of admissibility). When the $W_{i}$ represent pure states, then $S_{2}\left[W_{i}\right]=0$, and Eq. (18) becomes

$$
S_{2}[W] \leq 1-\sum_{i=1}^{N} \alpha_{i}^{2} .
$$

The equality sign holds when the $W_{i}$ are also orthogonal, as was shown in Sec. II.

2. Additivity. Let us consider two independent subsystems $A$ and $B$. The Wigner function $W$ describing the total system $A \cup B$ is simply given by the product of the Wigner functions $W_{A}$ and $W_{B}$ for the two subsystems

$$
W\left(x_{A}, p_{A}, x_{B}, p_{B}\right)=W_{A}\left(x_{A}, p_{A}\right) W_{B}\left(x_{B}, p_{B}\right) .
$$


It is easy to show that both the classical entropy, Eq. (四), and the Von Neumann entropy, Eq. (2), are additive [9], i.e. $S[W]=S\left[W_{A}\right]+S\left[W_{B}\right]$. This is a key property, since it enables one to identify the statistical entropy with the thermodynamical entropy, which is also additive.

By contrast, our definition of entropy is not additive in the usual sense. Let us first notice that, whereas the number of degrees of freedom of each subsystem is $D=1$, the total system has $D=2$. Therefore the information is defined as $I\left[W_{A, B}\right]=h \int W_{A, B}^{2}$ for each subsystem, and $I[W]=h^{2} \int W^{2}$ for the total system. With this in mind, it is easy to establish the following expression for the quantum information

$$
I[W]=I\left[W_{A}\right] I\left[W_{B}\right]
$$

which shows that, since $I<1$, the information contained in the total system is smaller than the information of each subsystem, except for pure states, for which $I=1$. In terms of the entropy $S_{2}=1-I$, Eq. (22) becomes

$$
S_{2}[W]=S_{2}\left[W_{A}\right]+S_{2}\left[W_{B}\right]-S_{2}\left[W_{A}\right] S_{2}\left[W_{B}\right] .
$$

The total entropy is therefore smaller than the sum of the partial entropies, but larger than each of them. Note that when the subsystems are "almost pure" quantum states, then $S_{2}\left[W_{A, B}\right] \ll 1$, and the non-additive correction to Eq. (23) becomes of higher order. In this case, approximate additivity is recovered.

It is also interesting to note that Eq. (23) is formally identical to the expression for the probability of the union of two subsets $A$ and $B$, which reads

$$
\operatorname{prob}(A \cup B)=\operatorname{prob}(A)+\operatorname{prob}(B)-\operatorname{prob}(A \cap B),
$$

and $\operatorname{prob}(A \cap B)=\operatorname{prob}(A) \operatorname{prob}(B)$ for statistically independent systems. The analogy of $S_{2}$ as probability is also consistent with the normalization $0 \leq S_{2} \leq 1$.

3. Subadditivity. If the subsystems $A$ and $B$ are not independent, the Wigner function cannot be factored as in Eq. (21). The Wigner function of each subsystem is then defined by integrating over the other system's variables, for instance

$$
W_{A}\left(x_{A}, p_{A}\right)=\int W\left(x_{A}, p_{A}, x_{B}, p_{B}\right) d x_{B} d p_{B},
$$

and similarly for $W_{B}$. For the Boltzmann-Von Neumann entropy, one can prove that $S[W] \leq$ $S\left[W_{A}\right]+S\left[W_{B}\right]$, and the equality sign holds when the two subsystems are independent [9]. This means that the total system $A \cup B$ contains more information than the sum of its parts - which is natural, since the two subsystems are correlated. However, no such relation can be proven for $S_{2}$ : this entropy is therefore not subadditive. Note that this fact is consistent with the analogy of $S_{2}$ as probability given by Eq. (24). Indeed, when the subsets $A$ and $B$ are not independent, the probability of their intersection $\operatorname{prob}(A \cap B)$ can be either smaller or larger than the product $\operatorname{prob}(A) \operatorname{prob}(B)$, corresponding to either negative or positive correlation.

4. Microcanonical Ensemble. We want to extremize the entropy $S_{2}$ with the constraint $\int W d x d p=1$. Using Lagrange multipliers, it is easy to show that the entropy is maximum 
when $W=$ const. $=\Omega^{-1}$ within a phase space region of volume (area) equal to $\Omega$, and $W=0$ elsewhere. In this case the entropy is

$$
S_{2}=1-\frac{h}{\Omega}, \quad(h=2 \pi \hbar) .
$$

This is the analog of Boltzmann's formula, Eq. (1), when the appropriate additive constant is used, i.e. $S_{\mathrm{B}}=\ln (\Omega / h)$. For both expressions, $S=0$ when $\Omega=h$ (minimum uncertainty), and the entropy becomes negative when $\Omega<h$, i.e. when the uncertainty relation is violated. In limit $\Omega \rightarrow \infty, S_{2}$ is bounded, and tends to unity (least information). With this notation, information $I=1-S_{2}$ is just the inverse of the number of available microstates $\Omega / h$.

5. Canonical Ensemble. We now extremize $S_{2}$ with the constraints $\int W d x d p=1$ and $\int W E d x d p=U$, where $E(x, p)=p^{2} / 2 m+\Phi(x)$, and $U$ is the average energy. Again using Lagrange multipliers, we find the following equilibrium distribution

$$
\begin{array}{llrl}
W_{\text {eq }}(x, p) & =Z^{-1}[1-\beta E(x, p)], & & \beta E<1 \\
W_{\text {eq }}(x, p) & =0, & & \beta E \geq 1
\end{array}
$$

where $\beta$ is the Lagrange multiplier corresponding to the energy constraint, and can be interpreted in the usual fashion as the inverse temperature $\beta=1 / T ; Z$ is a normalization constant. For energies such that $\beta E \ll 1$, Eq. (27) becomes identical with the standard exponential Boltzmann factor $\exp (-\beta E)$. Since $W_{\text {eq }}$ is a linear function of the energy, we have been forced to introduce a cut-off, otherwise $W_{\text {eq }}$ would diverge for large values of $E$. Physically, this means that states with energy $E>T$ are forbidden at equilibrium. Note the difference with standard thermodynamics, where such states are highly improbable (because Boltzmann's factor decreases exponentially), but not forbidden in principle.

An interesting fact is that Eq. (27) is a stationary solution of the Wigner equation (7) indeed, we are aware of no other stationary solution which is also a function of the energy $E(x, p)$ alone. This is easy to prove when the right-hand side of Eq. (7) is written as

$$
\sum_{n=0}^{\infty} c_{n} \frac{\partial^{2 n+1} \Phi}{\partial x^{2 n+1}} \frac{\partial^{2 n+1} W}{\partial p^{2 n+1}}
$$

where the $c_{n}$ are constants. The $n=0$ term yields the classical part of Wigner's equation, whereas all other terms do not provide any contribution, since $W_{\text {eq }}$ is quadratic in $p$. Moreover, since $W_{\text {eq }}$ is a function of the energy alone, it is a stationary solution of the classical Liouville equation, so that we have finally $\partial W_{\text {eq }} / \partial t=0$. The fact that maximizing the entropy $S_{2}$ naturally yields a Wigner function which is both stationary and a function of the energy alone is in itself remarkable. At the present stage, it is premature to make any statement about the role of $W_{\text {eq }}$, but the subject certainly deserves further attention. For example, it would be interesting to know if, and under what constraints, $W_{\text {eq }}$ can act as an attractor in a relaxation process.

\section{Smoothed Wigner Functions}

The Wigner function cannot be interpreted as a genuine probability distribution because it almost always takes negative values. The only pure state whose Wigner function is positive is given by the minimum uncertainty Gaussian wavepacket :

$$
\psi(x)=(2 \pi)^{-1 / 4} \sigma^{-1 / 2} \exp \left(-x^{2} / 4 \sigma^{2}\right),
$$


whose Wigner function is also Gaussian

$$
G(x, p)=\frac{1}{\pi \hbar} \exp \left(-\frac{x^{2}}{2 \sigma^{2}}-\frac{2 p^{2} \sigma^{2}}{\hbar^{2}}\right) .
$$

A possible way to obtain a positive distribution is to smooth a pure Wigner function $W(x, p)$ using a kernel $K(x, p)$ which is itself a Wigner function correponding to a pure state [10]. The smoothing operation is represented mathematically by a convolution in phase space. The smoothed Wigner function $\bar{W}(x, p)$

$$
\bar{W}(x, p)=\int W\left(x^{\prime}, p^{\prime}\right) K\left(x-x^{\prime}, p-p^{\prime}\right) d x^{\prime} d p^{\prime} \equiv W * K,
$$

is then positive and normalized to unity, so that it can be interpreted as a probability distribution.

In the past, the most common choice of the smoothing kernel has been the minimum uncertainty Gaussian $G(x, p)$, as given in Eq. (29) [10]. The resulting smoothed Wigner function is sometimes referred to as the Husimi function. This choice is however quite arbitrary, and no argument has ever been proposed, to our knowledge, in order to justify its privileged status. We shall now prove that smoothing with a Gaussian kernel does have some special properties, and should therefore be regarded as the correct way to obtain positive smoothed Wigner functions. In particular, it will be shown that, when the smoothing is performed with a Gaussian kernel, the result is still an admissible Wigner function.

First of all, we need a precise definition of an admissible Wigner function. Of course, not all functions of the phase space variables are admissible: for example, those functions which violate the uncertainty principle are clearly not admissible. Functions that can be constructed by summing orthogonal pure states, such as $W=\sum_{i} \alpha_{i} W_{i}$, are not admissible if some of the $\alpha_{i}$ are negative : this was the example analyzed in Sec. IV. Our definition of an admissible Wigner function is rather standard 传, and is based on the density matrix formalism. According to standard quantum theory, a density matrix $\rho$ must satisfy three properties in order to describe a quantum mixed state : (1) it must have unit trace $\operatorname{Tr} \rho=1$; (2) it must be Hermitian $\rho(x, y)=\rho^{*}(y, x)$; and (3) its eigenvalues must be non-negative. While the first two properties are easy to verify, the third is much harder to test, since one would need to diagonalize $\rho$ in order to compute its eigenvalues. Property (3) can also be expressed in the following way :

$$
\int \psi(x) \rho(x, y) \psi^{*}(y) d x d y \geq 0, \quad \forall \psi,
$$

where the inequality must hold for all wavefunctions $\psi$. This makes it even more apparent that Property (3) cannot be used as an operational test.

Now, the previous properties can be transposed to Wigner functions by making use of the definition, Eq. (5). In particular we would like to know whether the smoothed Wigner function $\bar{W}$ is in general admissible or not. Properties (1) and (2) simply require that $\bar{W}$ be real and normalized to unity. Property (3) can be written in the following form [4]

$$
\int \bar{W}(x, p) F(x, p) d x d p \geq 0, \quad \forall F(x, p)=\text { pure state } .
$$

The equivalence between Eqs. (31) and (32) can be verified by noting that $\bar{W}$ and $F$ are the Wigner transform of, respectively, $\rho$ and $\psi$, as defined in Eqs. (5-6). It is clear that, in 
order to check the admissibilty of $\bar{W}(x, p)$, one should perform an infinite number of integrals involving test Wigner functions $F(x, p)$ that represent pure states. However, Eq. (32) can be used to prove that smoothing with a Gaussian kernel yields a smoothed Wigner function which is itself admissible.

In order to do so, let us plug Eq. (30) into the left hand-side of Eq. (32). We obtain ( $W$ is the original Wigner function, $K$ is the smoothing kernel, and $F$ is the test function: all three represent pure states)

$$
\begin{aligned}
& \int W\left(x-x^{\prime}, p-p^{\prime}\right) F(x, p) K\left(x^{\prime}, p^{\prime}\right) d x^{\prime} d p^{\prime} d x d p \\
& =\int K\left(x^{\prime}, p^{\prime}\right) d x^{\prime} d p^{\prime} \int W_{1}\left(x-x^{\prime}, p-p^{\prime}\right) F(x, p) d x d p \\
& =\int K\left(x^{\prime}, p^{\prime}\right)\left[W_{1} * F\right]\left(x^{\prime}, p^{\prime}\right) d x^{\prime} d p^{\prime},
\end{aligned}
$$

where $W_{1}(x, p)=W(-x,-p)$ is the Wigner function corresponding to the wavefunction $\psi(-x)$ [whereas $W$ corresponds to $\psi(x)$ ]. The term $W_{1} * F$ is certainly a positive function, since it is the convolution product of two Wigner functions. It follows that a sufficient condition for Eq. (32) to be satisfied is that $K(x, p)$ be positive. But the only pure state Wigner function which is also positive is the Gaussian $G(x, p)$ [Eq. (29)]. This proves that, when the smoothing kernel is Gaussian, the inequality given in Eq. (32) is verified, and the smoothed Wigner function $\bar{W}(x, p)$ is therefore admissible. In this case, the density matrix $\bar{\rho}$ corresponding to $\bar{W}$ can be written as

$$
\bar{\rho}(x, y)=\frac{1}{\sqrt{2 \pi} \sigma} \int W(q, p) \exp \left(-\frac{(x-q)^{2}}{4 \sigma^{2}}+\frac{i p x}{\hbar}\right) \exp \left(-\frac{(y-q)^{2}}{4 \sigma^{2}}-\frac{i p y}{\hbar}\right) d q d p .
$$

The previous result can be easily checked by computing the Wigner function $\bar{W}$ associated to $\bar{\rho}$ via Eq. (5), and realizing that it can be written as $\bar{W}=W * G$. Equation (34) expresses the density matrix as a continuous sum of localized states in phase space ('coherent states' [11]). Note that the coefficients in this sum [i.e. $W(x, p)$ itself] are not necessarily positive numbers. The reason for this is that the set of coherent states is 'overcomplete', meaning that the representation of an arbitrary quantum state in terms of coherent states is not unique. However, thanks to the previous theorem, we know that a diagonal representation of $\bar{\rho}$ with non-negative coefficients does exist, although we are not generally able to construct it explicitly.

So far we have proven that smoothing with a Gaussian kernel yields a function $\bar{W}$ which is itself an admissible Wigner function. Nothing definite can be said when the smoothing is performed using a different kernel. However, we are able to produce a counterexample, i.e. a pure state Wigner function which, after smoothing with a non-Gaussian kernel, does not satisfy Eq. (32), and is therefore not admissible. Let us consider the wavefunction

$$
\psi(x)=2(2 / \pi)^{1 / 4} x \exp \left(-x^{2}\right),
$$

and call $W(x, p)$ its Wigner transform. Now we smooth $W$ using as kernel $W$ itself:

$$
\bar{W}=W * W .
$$

In order to be an admissible Wigner function, $\bar{W}$ must satisfy Eq. (32) for every test function $F$. Let us use as test function once again $W$ itself, and compute the integral in Eq. (32). We obtain (details are in the Appendix)

$$
\int \bar{W}(x, p) W(x, p) d x d p=-\frac{1}{27 \pi \hbar}<0 .
$$


This result shows that not all ways of smoothing Wigner functions are equivalent: only by smoothing with a Gaussian kernel we are certain to obtain a function which is positive and also represents an admissible quantum state (i.e. a state defined by a density matrix with real non-negative eigenvalues).

Furthermore, Eq. (33) suggests another way to construct a phase space distribution which is both positive and admissible [satisfying Eq. (32)]. Let us take for $W(x, p)$ an arbitrary positive function of phase space variables, and smooth it with a Gaussian kernel $G(x, p)$ : $\bar{W}=W * G$. We want to prove that $\bar{W}$ is admissible. Equation (32) yields (using the fact that $G$ is even)

$$
\begin{aligned}
& \int W\left(x-x^{\prime}, p-p^{\prime}\right) F(x, p) G\left(x^{\prime}, p^{\prime}\right) d x^{\prime} d p^{\prime} d x d p \\
& =\int W\left(x^{\prime}, p^{\prime}\right) d x^{\prime} d p^{\prime} \int G\left(x^{\prime}-x, p^{\prime}-p\right) F(x, p) d x d p \\
& =\int W\left(x^{\prime}, p^{\prime}\right)[G * F]\left(x^{\prime}, p^{\prime}\right) d x^{\prime} d p^{\prime}>0 .
\end{aligned}
$$

The result follows from the fact that the convolution product is positive, since both $F$ and $G$ are pure state Wigner functions, and $W>0$ because we chose it to be so. This proves that $\bar{W}(x, p)$ is an admissible Wigner function, and is also positive, since it is the convolution product of two positive functions. The density matrix corresponding to $\bar{W}$ is again $\bar{\rho}$, as given by Eq. (34). Physically, the smoothed function $\bar{W}=W * G$ can be interpreted as the admissible quantum state which best approximates the classical state $W$ for a given value of $\hbar$.

To conclude this Section, we restate the two main results that have been obtained here. We have shown two possible ways to construct a phase space distribution which is both positive and an admissible quantum state. This can be performed $(a)$ by smoothing a pure state Wigner function with a Gaussian kernel, or (b) by smoothing an arbitrary (but positive) function of phase space variables, again with a Gaussian kernel. Therefore, the Gaussian function $G(x, p)$ given in Eq. (29) has a privileged status as a smoothing kernel. Note, however, that $G$ is not unique, since it depends on the parameter $\sigma$.

Although such results were derived for a pure state Wigner function, they can easily be generalized to mixtures. It follows that, when smoothing several times with a Gaussian kernel, we still remain within the class of admissible Wigner functions. This class is therefore closed with respect to this particular operation.

\section{Entropy and Smoothed Wigner Functions}

The smoothing operation has the effect of erasing some of the correlations in the phase space. We expect therefore that smoothing should increase the entropy. This is not difficult to prove. In order to do this, we need to define the double Fourier transform of a Wigner function $W(x, p)$

$$
W(k, \lambda)=\iint W(x, p) \exp (-i k x-i \lambda p) d x d p .
$$

By means of Eqs. (16) and (39), one obtains for a pure state

$$
W(k, \lambda)=\int \psi\left(x-\frac{\lambda \hbar}{2}\right) \psi^{*}\left(x+\frac{\lambda \hbar}{2}\right) \exp (-i k x) d x .
$$

We can then easily prove the following Lemma

$$
|W(k, \lambda)|^{2} \leq \int\left|\psi\left(x-\frac{\lambda \hbar}{2}\right) d x\right|^{2} \int\left|\psi^{*}\left(x+\frac{\lambda \hbar}{2}\right) d x\right|^{2}=1
$$


where use has been made of Schwartz's inequality.

Now, let us take an arbitrary Wigner function $W(x, p)$ and smooth it with a kernel $K(x, p)$ which is a pure state: $\bar{W}(x, p)=W(x, p) * K(x, p)$. In Fourier space we have : $\bar{W}(k, \lambda)=$ $W(k, \lambda) K(k, \lambda)$. The quantum information $I[\bar{W}]=2 \pi \hbar \int \bar{W}^{2} d x d p$ relative to $\bar{W}$ satisfies the inequalities

$$
\begin{aligned}
& I[\bar{W}]=\frac{\hbar}{2 \pi} \int|\bar{W}(k, \lambda)|^{2} d k d \lambda=\frac{\hbar}{2 \pi} \int|W(k, \lambda)|^{2}|K(k, \lambda)|^{2} d k d \lambda \\
& \leq \max |K(k, \lambda)|^{2} \times \frac{\hbar}{2 \pi} \int|W(k, \lambda)|^{2} d k d \lambda \\
& =\max |K(k, \lambda)|^{2} \times 2 \pi \hbar \int W^{2} d x d p \leq 2 \pi \hbar \int W^{2} d x d p=I[W]
\end{aligned}
$$

where we have used the previous Lemma [Eq. (41)] for $K$, as well as Parseval's identity in the form

$$
\int W^{2}(x, p) d x d p=\frac{1}{4 \pi^{2}} \int|W(k, \lambda)|^{2} d k d \lambda .
$$

Equation (42) implies that

$$
S_{2}[\bar{W}] \geq S_{2}[W],
$$

i.e. the smoothing operation has increased the entropy. Note that, in order to obtain this result, the smoothing kernel needs not be a Gaussian.

Now we turn to the case where the smoothing kernel is indeed Gaussian. In this case, a relatively simple expression for $I[\bar{W}]$ can be obtained. The double Fourier transform of the Gaussian defined in Eq. (29) is

$$
G(k, \lambda)=\exp \left(-\frac{k^{2} \sigma^{2}}{2}-\frac{\lambda^{2} \hbar^{2}}{8 \sigma^{2}}\right) .
$$

The Fourier transform of the Wigner function $W$ to be smoothed is given by Eq. (40). Let us compute the information :

$$
I[\bar{W}]=2 \pi \hbar \int \bar{W}^{2} d x d p=\frac{\hbar}{2 \pi} \int|W(k, \lambda)|^{2}|G(k, \lambda)|^{2} d k d \lambda .
$$

Expressing $W$ and $G$ by means of Eqs. (40), (45) one obtains, after some algebra

$$
\begin{gathered}
I[\bar{W}]=\frac{1}{2 \sigma \sqrt{\pi}} \int \psi\left(x-\frac{\lambda}{2}\right) \psi^{*}\left(x+\frac{\lambda}{2}\right) \psi\left(x^{\prime}+\frac{\lambda}{2}\right) \times \\
\psi^{*}\left(x^{\prime}-\frac{\lambda}{2}\right) \exp \left(-\frac{\lambda^{2}+\left(x-x^{\prime}\right)^{2}}{4 \sigma^{2}}\right) d x d x^{\prime} d \lambda .
\end{gathered}
$$

We now change the integration variables, using the following unitary transformation

$$
\begin{aligned}
& x=\frac{1}{2} w+\frac{1}{2} y+\frac{1}{2} z \\
& x^{\prime}=\frac{1}{2} w-\frac{1}{2} y-\frac{1}{2} z \\
& \lambda=-y+z .
\end{aligned}
$$

After some algebra, the following result is obtained

$$
I[\bar{W}]=\frac{1}{\sigma \sqrt{\pi}} \int d w\left|\int \psi(w+y) \psi(w-y) \exp \left(-\frac{y^{2}}{2 \sigma^{2}}\right) d y\right|^{2}
$$


which expresses the quantum information in terms of the wavefunction $\psi$ corresponding to the unsmoothed Wigner function $W$. Equation (49) may be usefully employed to monitor the time evolution of the entropy in a numerical simulation: $\psi(x, t)$ would then evolve according to the time-dependent Schrödinger equation.

Finally, we show that a more stringent bound than the one expressed by Eq. (44) can be obtained when the smoothing kernel is Gaussian. By using again Schwartz's inequality, we have from Eq. (49)

$$
\begin{gathered}
I[\bar{W}] \leq \frac{1}{\sigma \sqrt{\pi}} \int d w \int d y \exp \left(-\frac{y^{2}}{\sigma^{2}}\right) \int d y^{\prime}\left|\psi\left(w+y^{\prime}\right) \psi\left(w-y^{\prime}\right)\right|^{2} \\
=\int d w \int d y^{\prime}\left|\psi\left(w+y^{\prime}\right) \psi\left(w-y^{\prime}\right)\right|^{2}=\frac{1}{2} .
\end{gathered}
$$

In terms of the entropy, this becomes

$$
S_{2}[\bar{W}] \geq \frac{1}{2}
$$

a result that is valid when smoothing a pure Wigner function with a Gaussian kernel. Note that we still have some freedom in the choice of the kernel, since the width $\sigma$ of the Gaussian in Eq. (29) is still unspecified. It would be interesting, for example, to know which value of $\sigma$ minimizes the entropy $S_{2}[\bar{W}]$, within the bounds given by Eq. (51). We have not been able to obtain a general result, but some indication can be obtained from the following example. Let us suppose that the function $W$ to be smoothed is also a Gaussian, as in Eq. (29), but with spatial variance $\mu$ instead of $\sigma$. The smoothed Wigner function is then

$$
\bar{W}(x, p)=W * G=\frac{1}{2 \pi \Sigma_{x} \Sigma_{p}} \exp \left(-\frac{x^{2}}{2 \Sigma_{x}^{2}}-\frac{p^{2}}{2 \Sigma_{p}^{2}}\right)
$$

with

$$
\Sigma_{x}^{2}=\sigma^{2}+\mu^{2} \quad ; \quad \Sigma_{p}^{2}=\frac{\hbar^{2}}{4}\left(\frac{1}{\sigma^{2}}+\frac{1}{\mu^{2}}\right)
$$

The information corresponding to $\bar{W}$ is

$$
I[\bar{W}]=2 \pi \hbar \int \bar{W}^{2} d x d p=\frac{\hbar}{2 \Sigma_{x} \Sigma_{p}} .
$$

After some algebra, one obtains the following expression

$$
I[\bar{W}]=I(z)=\frac{z}{1+z^{2}},
$$

where $z=\sigma / \mu$. The function $I(z)$ attains its maximum for $z=1$, i.e. when $\sigma=\mu$, and the kernel has the same variance as the Wigner function to be smoothed. In this case, $S_{2}[\bar{W}]=1 / 2$, which represents the lower bound of Eq. (51). We could conjecture, although we do not have a formal proof, that this is the general result : the minimum entropy increase due to smoothing with a Gaussian kernel is attained when the width of the kernel is close to the width of the function to be smoothed.

Another interesting example is provided by the harmonic oscillator, whose Hamiltonian is

$$
H(x, p)=\frac{p^{2}}{2 m}+m \omega^{2} \frac{x^{2}}{2} .
$$


The eigenstates can be expressed in terms of Hermite polynomials $H_{n}(\xi)\left(H_{0}=1, H_{1}=\right.$ $\left.2 \xi, H_{2}=4 \xi^{2}-2, \ldots\right)$

$$
\psi_{n}(x)=\left(2^{n} n !\right)^{-1 / 2}\left(\frac{m \omega}{\hbar \pi}\right)^{1 / 4} \exp \left(-\frac{m \omega x^{2}}{2 \hbar}\right) H_{n}(x \sqrt{m \omega / \hbar}) .
$$

The corresponding Wigner functions are [3]

$$
W_{n}(x, p)=\frac{(-1)^{n}}{\pi \hbar} \exp \left(-\frac{2 H}{\hbar \omega}\right) L_{n}\left(\frac{4 H}{\hbar \omega}\right)
$$

where $H(x, p)$ is the Hamiltonian, and the $L_{n}(\xi)$ are Legendre polynomials $\left(L_{0}=1, L_{1}=\right.$ $\left.1-\xi, L_{2}=1-2 \xi+\xi^{2} / 2, \ldots\right)$. We now smooth such Wigner functions with a Gaussian kernel, and find 3$]$

$$
\bar{W}_{n}(x, p)=(2 \pi \hbar n !)^{-1}(H / \hbar \omega)^{n} \exp (-H / \hbar \omega) .
$$

Note that this relatively simple result for $\bar{W}_{n}$ is obtained only in the case when the square variance of the smoothing kernel [see Eq. (29)] is $\sigma^{2}=\hbar / 2 m \omega$; in all other cases the smoothed Wigner function is not a function of the energy only. We are now in a position to compute the information $I\left[\bar{W}_{n}\right] \equiv \bar{I}_{n}=2 \pi \hbar \int \bar{W}_{n}^{2} d x d p$. Let us first change to polar coordinates $(r, \theta)$ in the phase space

$$
\frac{p^{2}}{m}+m \omega^{2} x^{2}=\hbar \omega r^{2}, \quad d x d p=\hbar r d r d \theta .
$$

One obtains, after integration over $\theta$

$$
\bar{I}_{n}=(n !)^{-2} \int_{0}^{\infty}\left(r^{2} / 2\right)^{2 n} \exp \left(-r^{2}\right) r d r
$$

and finally, changing variable again $z=r^{2} / 2$

$$
\bar{I}_{n}=(n !)^{-2} \int_{0}^{\infty} z^{2 n} \exp (-2 z) d z=\frac{(2 n) !}{2^{2 n+1}(n !)^{2}} .
$$

We first note that $\bar{I}_{0}=1 / 2$, in agreement with previous results, since the ground state of the harmonic oscillator is a Gaussian, and we are smoothing with another Gaussian of identical width. It can also be shown that $\bar{I}_{n}$ is a decreasing function of $n$. The asymptotic expansion (for $n \gg 1$ ) is obtained by taking the logarithm of Eq. (61) and making use of Stirling's formula

$$
\ln X ! \sim X \ln X-X+\frac{1}{2} \ln X \quad(X \gg 1)
$$

which yields

$$
\bar{I}_{n} \sim n^{-1 / 2} .
$$

In terms of the entropy, we have in summary

$$
\begin{aligned}
& S_{2}\left[\bar{W}_{0}\right]=1 / 2 \\
& S_{2}\left[\bar{W}_{n+1}\right]>S_{2}\left[\bar{W}_{n}\right] \\
& \lim _{n \rightarrow \infty} S_{2}\left[\bar{W}_{n}\right]=1 .
\end{aligned}
$$

The latter results means that the entropy increase is larger when smoothing a semi-classical state. Asymptotically, the entropy of the smoothed Wigner function approaches unity. On the other hand, when smoothing a 'fully quantum' state (i.e. a state with small quantum numbers), the entropy increase is moderate. Although these results were obtained for the 
special case of the harmonic oscillator, we are confident that they remain qualitatively correct for other (classically integrable) Hamiltonians.

\section{Discussion}

In this paper we have presented several results related to a new definition of quantum entropy, denoted $S_{2}$. Although it has already been used in the past in the framework of the density matrix formalism, such entropy becomes particularly interesting when applied to Wigner functions. It is then possible to show that $S_{2}$ possesses a number of interesting properties - most importantly, for example, it is an invariant for the Wigner equation, which governs the evolution of Wigner functions. $S_{2}$ is related to the Tsallis entropy, although the latter is usually defined for a discrete set of probabilities, rather than for a continuous distribution. An advantage of this entropy, compared to the quantum Von Neumann entropy, is that the Wigner function is all that one needs to compute $S_{2}$. No knowledge of the density matrix is required, neither does it need to be diagonalized, as is the case for the Von Neumann entropy.

The standard properties of entropy (concavity, additivity, sub-additivity) have been examined. This has revealed some interesting facts, which would require further investigations. For instance, it has been proven that $S_{2}$ (unlike ordinary entropy) behaves like a probabilty with respect to additivity properties, which is also consistent with the normalization $0 \leq S_{2} \leq 1$. Secondly, the analysis of the canonical ensemble has enabled us to derive a Wigner function $W_{\text {eq }}$ that maximizes the entropy under certain constraints. $W_{\text {eq }}$ turns out to be both a function of the energy alone and a stationary solution of the Wigner equation. The relevance of $W_{\text {eq }}$ is still unclear, but one could reasonably conjecture that it plays a role in some relaxation processes. Numerical experiments could clarify this point.

An "unpleasant" property of $S_{2}$ is that, keeping the Wigner function fixed, and letting Planck's constant go to zero, one obtains $S_{2}=1$. Thus it would seem that all classical states have unit entropy. The point is that this is not the correct procedure to obtain a classical state: indeed, if the original Wigner function is negative somewhere, we would obtain a classical state with a non-positive probability distribution, which is of course meaningless. The correct procedure is instead to smooth the Wigner function $W$ with an appropriate kernel, which must also be a Wigner function in order to ensure positivity. A crucial point, however, is that the smoothed Wigner function $\bar{W}$ should be itself an admissible quantum state, i.e. one that can be described by a density matrix with non-negative eigenvalues. We have been able to prove that, when smoothing with a minimum uncertainty Gaussian packet, the result is always admissible - although this is not necessarily the case when smoothing with another Wigner function. This is, to our knowledge, the first rigorous argument showing that Gaussian smoothing possesses some privileged status.

It has also been proven that smoothing increases the entropy: in particular, when smoothing a pure state with a Gaussian kernel, one has $S_{2}[\bar{W}] \geq 1 / 2$. It would be interesting to know how to minimize $S_{2}[\bar{W}]$. This could be done by varying the width $\sigma$ of the Gaussian kernel, which is still a free parameter. Although we are not able to derive a rigorous risult, we have conjectured (and shown explicitly on a particular example) that $S_{2}[\bar{W}]$ is minimum when the width of the Gaussian kernel is close to the width of the Wigner function to be smoothed. This would not be unreasonable from the information point of view : it would mean that we can minimize the entropy increase if we have some prior knowledge of the function to be smoothed. 
As a further example, we have computed the entropy of the (smoothed) stationary states of the harmonic oscillator. It was shown that $S_{2}$ increases with quantum number, therefore semi-classical states yield a larger entropy increase. Again, we have conjectured that this behavior is universal (at least for confining and classically integrable Hamiltonians), and not specific to the harmonic oscillator. We are rather confident that our conjecture is correct since the larger entropy increase for semi-classical states is mainly due to the fact that their Wigner function displays short-wavelength oscillations in the phase space, which are easily erased by the smoothing procedure.

It would be interesting to know how the previous results generalizes to classically nonintegrable Hamiltonians. For the harmonic oscillator, it was found that the information of the smoothed stationary states behaves as $\bar{I}_{n} \sim n^{-1 / 2}$. Although the exponent $-1 / 2$ might be specific to the harmonic oscillator, a polynomial law may be universal for the class of integrable Hamilationans. On the other hand, one could conjecture that, for non-integrable Hamiltonians, the decrease is faster, perhaps exponential.

From the physical point of view, this result means that semi-classical states are highly unstable under generic perturbations (amongst which smoothing is a relevant example). This is reminiscent of the so-called 'predictability sieve', a concept introduced by W.H. Zurek and coworkers [5] in the more general framework of decoherence [12, 13]. Zurek et al. [5] construct a model for the interaction of a quantum system with an environment at thermodynamic equilibrium, and compute the rate at which initially pure states deteriorate into mixtures by coupling with the environment. This process is known as decoherence. Subsequently, they look for the set of states which are least prone to deterioration, and find that such states are those which yield the minimum entropy increase. By estimating the entropy production, they obtain that the minimum-entropy increase is attained for the ground state of the harmonic oscillator, i.e. a minimum uncertainty Gaussian wavepacket. This coincides with our results of Sec. V.

The main difference from our approach is that W.H. Zurek and co-workers [5] analyze a dynamical situation, while in our case the entropy-producing effect is the smoothing, which is a static process. Since both cases appear to give the same result, it is reasonable to conjecture that smoothing may represent a (simplified) model for the interaction of a quantum system with an open environment. The price to pay for our approach is that we do not have a first-principle based derivation of such an interaction. The advantage is that the model is simple enough to obtain a number of rigorous results.

These considerations may shed some new light on the semi-classical limit. We distinguish two kinds of pure quantum states: fully quantum (FQ) states $W_{\mathrm{FQ}}$ (with low quantum numbers), and semi-classical (SC) states $W_{\mathrm{SC}}$ (with large quantum numbers). For both $S_{2}=0$, i.e. they contain the same amount of information. However, after the smoothing, one obtains $S_{2}\left[\bar{W}_{\mathrm{FQ}}\right] \simeq 1 / 2$ and $S_{2}\left[\bar{W}_{\mathrm{SC}}\right] \rightarrow 1$, i.e. the smoothed FQ state contains more information than the smoothed SC state. In other words, although both original states contain the same information, this is of different 'quality': robust for the FQ state, and highly prone to deterioration for the $\mathrm{SC}$ state. It is not surprising, therefore, that coupling to an environment has the effect of erasing such information less easily in the former case than in the latter. These results could open new avenues for further research, particularly with computer experiments [14], to investigate the dynamical behavior of the entropy defined in this paper. 


\section{Appendix}

We want to prove the result of Eq. (37). Let us use the identity

$$
\int f(x, p) g(x, p) d x d p=\frac{1}{4 \pi^{2}} \int f(k, \lambda) g^{*}(k, \lambda) d k d \lambda,
$$

with $f=\bar{W}$ and $g=W$. Since $\bar{W}(x, p)=W * W$, the double Fourier transform of $\bar{W}$ is $W^{2}(k, \lambda)$. In addition, it will turn out that $W(k, \lambda)$ is real for the case under consideration here. Therefore, by making use of the previous identity, the left-hand side of Eq. (37) becomes

$$
\int \bar{W}(x, p) W(x, p) d x d p=\frac{1}{4 \pi^{2}} \int W^{3}(k, \lambda) d k d \lambda
$$

The double Fourier transform $W(k, \lambda)$ is given by Eq. (40). For our example, the wavefunction is the one of Eq. (35), and we obtain

$$
W(k, \lambda)=4 \sqrt{2 / \pi} \exp \left(-\lambda^{2} \hbar^{2} / 2\right) \int\left(x^{2}-\frac{\lambda^{2} \hbar^{2}}{4}\right) \exp \left(-2 x^{2}\right) \exp (-i k x) d x
$$

Now, by using the following integrals

$$
\begin{gathered}
\int \exp \left(-2 x^{2}\right) \exp (-i k x) d x=\sqrt{\frac{\pi}{2}} \exp \left(-k^{2} / 8\right) \\
\int x^{2} \exp \left(-2 x^{2}\right) \exp (-i k x) d x=\sqrt{\frac{\pi}{8}}\left(1-\frac{k^{2}}{4}\right) \exp \left(-k^{2} / 8\right),
\end{gathered}
$$

we obtain, after some straightforward algebra

$$
W(k, \lambda)=\left(1-\frac{k^{2}}{4}-\lambda^{2} \hbar^{2}\right) \exp \left(-\frac{\lambda^{2} \hbar^{2}}{2}-\frac{k^{2}}{8}\right) .
$$

We are now ready to compute the integral $\int W^{3} d k d \lambda$. Let us change integration variables $(k, \lambda) \rightarrow(r, \varphi)$

$$
r^{2}=\frac{k^{2}}{4}+\lambda^{2} \hbar^{2} \quad ; \quad r d r d \varphi=\frac{\hbar}{2} d \lambda d k .
$$

After integration over $\varphi$, one obtains

$$
\int_{-\infty}^{\infty} \int_{-\infty}^{\infty} W^{3} d k d \lambda=\frac{4 \pi}{\hbar} \int_{0}^{\infty}\left(1-r^{2}\right)^{3} \exp \left(-\frac{3}{2} r^{2}\right) r d r
$$

Changing the integration variable to $y=r^{2}$ and using integrals of the type

$$
\int_{0}^{\infty} y^{n} \exp (-a y) d y=\frac{n !}{a^{n+1}}
$$

it is obtained

$$
\int_{-\infty}^{\infty} \int_{-\infty}^{\infty} W^{3} d k d \lambda=\frac{2 \pi}{\hbar} \int_{0}^{\infty}(1-y)^{3} \exp \left(-\frac{3}{2} y\right) d y=-\frac{4 \pi}{27 \hbar}
$$

which, once divided by $4 \pi^{2}$, yields the result of Eq. (37). 


\section{References}

[1] John Von Neumann, The Mathematical Foundations of Quantum Mechanics (Princeton University Press, Princeton, 1955).

[2] E.P. Wigner, Phys. Rev. 40, 749 (1932).

[3] V.I. Tatarskii, Usp. Fiz. Nauk 139, 587 (1983) [Sov. Phys. Usp. 26, 311 (1983)].

[4] M. Hillery, R.F. O'Connell, M.O. Scully, and E.P. Wigner, Phys. Rep. 106, 121 (1984).

[5] W.H. Zurek, S. Habib, and J.P. Paz, Phys. Rev. Lett 70, 1187 (1993).

[6] A.K. Pattanayak, Phys. Rev. Lett 83, 4526 (1999).

[7] C. Tsallis, J. Stat. Phys. 52, 479 (1988).

[8] Bruce M. Boghosian, Phys. Rev. E 53, 4754 (1996).

[9] A. Wehrl, Rev. Mod. Phys. 50, 221 (1978).

[10] K. Husimi, Proc. Phys. Math. Soc. Japan 22, 264 (1940); N.D. Cartwright, Physica 83A, 210 (1976).

[11] R.J. Glauber, Phys. Rev. Lett 10, 84 (1963); S. Howard and S.K. Roy, Am. J. Phys. 55, 1109 (1987).

[12] W.H. Zurek, Phys. Rev. D 24, 1516 (1981); Phys. Rev. D 26, 1862 (1982); Phys. Today 44, No. 10, 36 (1991).

[13] A.K. Pattanayak and P. Brumer, Phys. Rev. Lett 79, 4131 (1997); S. Habib, K. Shizume, and W.H. Zurek, Phys. Rev. Lett 80, 4361 (1998); J.P. Paz and W.H. Zurek, Phys. Rev. Lett 82, 5181 (1999).

[14] G. Manfredi and M.R. Feix, in Advances in Kinetic Theory and Computing, edited by B. Perthame (World Scientific, Singapore, 1994), pp. 109-140; G. Manfredi and M.R. Feix, Phys. Rev. E 53, 6460 (1996). 